the narrow aortic annulus and that the development of CPF may have been promoted.

In this case, endocarditis was initially suspected because of vegetation-like mobile masses that were found in the aortic valve on the transesophageal echocardiogram, but infection was negative in all other examination findings. We determined before surgery that these masses were most likely cardiac tumors. Mild exertional dyspnea was the only clinical symptom, but it is known that mobile tumors attached to an aortic valve can cause serious complications such as thromboembolism and myocardial ischemia, ${ }^{4}$ and we decided that a surgical excision was necessary.

QAV with a narrow aortic annulus presenting with the development of multiple CPFs is a rare complication. There have been no similar cases reported in the literature. However, according to the characteristics of each pathologic condition, there is a possibility that CPF may develop with QAV.

\section{References}

1. Croccia MG, Chiaramonti F, Pratali S, Scioti G, Guarracino F, Bortolotti U. Quadricuspid aortic valve associated with hypoplastic aortic annulus. J Thorac Cardiovasc Surg. 2007;134:1061-2.

2. Hurwitz LE, Roberts WC. Quadricuspid semilunar valves. Am J Cardiol. 1973;31:623-6.

3. Klarich KW, Enriquez-Sarano M, Gura GM, Edwards WD, Tajik AJ, Seward JB. Papillary fibroelastoma: echocardiographic characteristics for diagnosis and pathologic correlation. J Am Coll Cardiol. 1997;30:784-90.

4. Jobic Y, Etienne Y, Quintin-Roué I, Dewilde J, Cornec P, Gilard M, et al. Left ventricular papillary fibroelastoma: two-dimensional echocardiographic detection and surgical resection. J Am Soc Echocardiogr. 1995;8:756-8.

\title{
Pulmonary venous obstruction in a patient with Marfan syndrome: Rare presentation of an expanding dissecting descending thoracic aortic aneurysm with annuloaortic ectasia
}

Yoshikatsu Saiki, MD, PhD, Shunsuke Kawamoto, MD, PhD, Junetsu Akasaka, MD, PhD, Naotaka Motoyoshi, MD, PhD, Sadahiro Sai, MD, PhD, and Koichi Tabayashi, MD, PhD, Sendai, Japan

Pulmonary venous obstruction (PVO) is an extremely rare condition in patients with acquired cardiovascular disease. We describe the case of a patient with chronic dissecting descending thoracic aortic aneurysm and annuloaortic ectasia associated with Marfan syndrome in whom PVO developed. The patient underwent successful emergency single-stage repair of the extensive thoracic aortic aneurysm.

\section{CLINICAL SUMMARY}

A 15-year-old female patient with Marfan syndrome had been followed up at a local hospital for annuloaortic ectasia with mild aortic insufficiency. A type B acute aortic dissection developed and was managed conservatively. Four months later, the patient had sudden onset of severe back pain, and computed tomographic (CT) scans revealed expansion of the dissecting descending thoracic aorta. She

From the Department of Cardiovascular Surgery, Graduate School of Medicine, Tohoku University, Sendai, Japan.

Received for publication Dec 23, 2007; accepted for publication Jan 12, 2008.

Address for reprints: Yoshikatsu Saiki, MD, Department of Cardiovascular Surgery, Graduate School of Medicine, Tohoku University, Seiryomachi, Aoba-ku, Sendai,

Japan 980-8574 (E-mail: ysaiki@mail.tains.tohoku.ac.jp).

J Thorac Cardiovasc Surg 2009;137:1282-4

0022-5223/\$36.00

Copyright $@ 2009$ by The American Association for Thoracic Surgery doi:10.1016/j.jtcvs.2008.01.043 was admitted to our intensive care unit because rupture of the thoracic aorta appeared impending. Sixteen hours after admission, large amounts of foamy sputum were observed. Repeated chest radiographs revealed pulmonary venous congestion, specifically on the right side (Figure 1, A). A transthoracic echocardiogram revealed a moderate degree of aortic regurgitation and turbulent right pulmonary venous flow. On the basis of the CT scans, the PVO on the right side was attributed to compression by three anatomic components: the expanding dissecting descending thoracic aortic aneurysm, the annuloaortic ectasia, and the deformed thorax (Figure 1, B). Emergency surgery was undertaken to repair the extensive disease of the thoracic aorta and to resolve the PVO.

The patient was placed in a semidecubitus position with her left side facing up. Prosthetic vascular grafts $(6 \mathrm{~mm})$ were anastomosed to the sides of the right subclavian, left axillary, and left femoral arteries, for arterial inflow. Bilateral anterior thoracotomy through the fourth intercostal space with limited incision on the right side, transverse sternotomy, transection of the fifth and sixth ribs, and further extension of the sixth intercostal incision were used to expose the entire thoracic aorta and heart (Figure 2, A). Aortic root replacement with a composite graft with a bileaflet mechanical valve was performed under conditions of hypothermic 

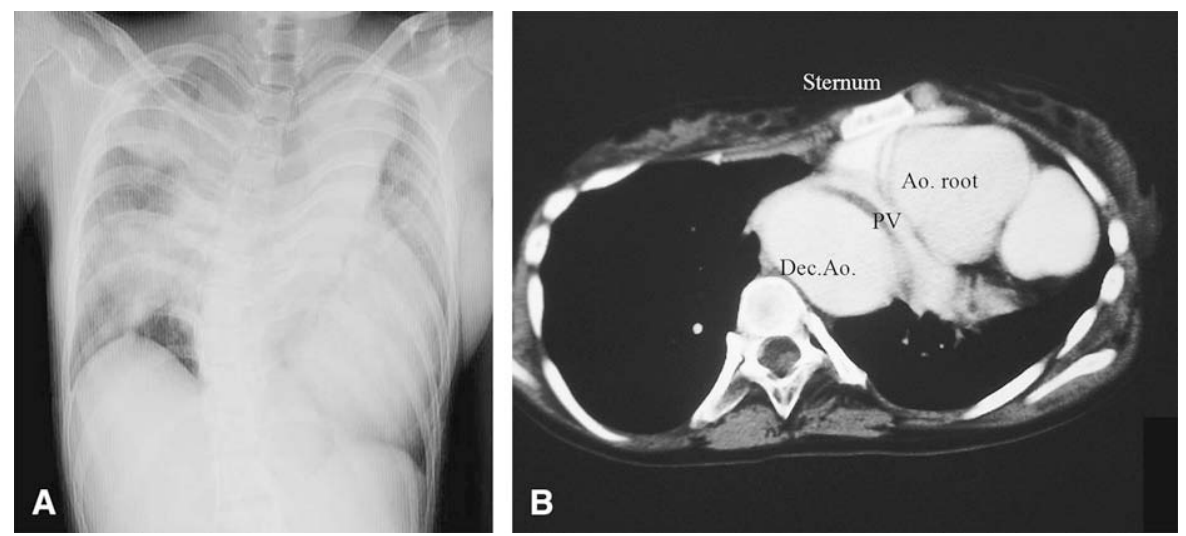

FIGURE 1. Preoperative imaging. A, Chest radiograph showing severe congestion in right lung. B, Computed tomographic scan of left atrial region showing right pulmonary vein being compressed by surrounding components: dissecting descending thoracic aorta, enlarged aortic root, and deformed chest wall. Ao. Root, Aortic root; $P V$, pulmonary vein; Dec. Ao., descending thoracic aorta.

cardiopulmonary bypass. After establishment of selective cerebral perfusion by direct cannulation into the left carotid artery, the descending thoracic aorta was crossclamped at the level below the tenth intercostal artery and then incised. Blood flow from the orifices of the intercostal arteries was controlled with several A-shield occlusion catheters (Asahi Intec Co Ltd, Seto, Japan). Distal anastomosis between a handmade composite graft and the descending thoracic aorta was carried out in a double-barrel fashion because of the narrow true lumen. The eighth, ninth, and tenth intercostal arteries were reattached with $10-\mathrm{mm}$ side branches of the composite graft. A separate 8-mm graft was anastomosed to the root of the left subclavian artery to facilitate subsequent reconstruction. A four-branched prosthetic graft was used for the arch graft. The arch vessels were serially reconstructed during rewarming with antegrade perfusion through a 10-mm sidearm graft (Figure 2, B). Weaning from cardiopulmonary bypass was uneventful. The patient was
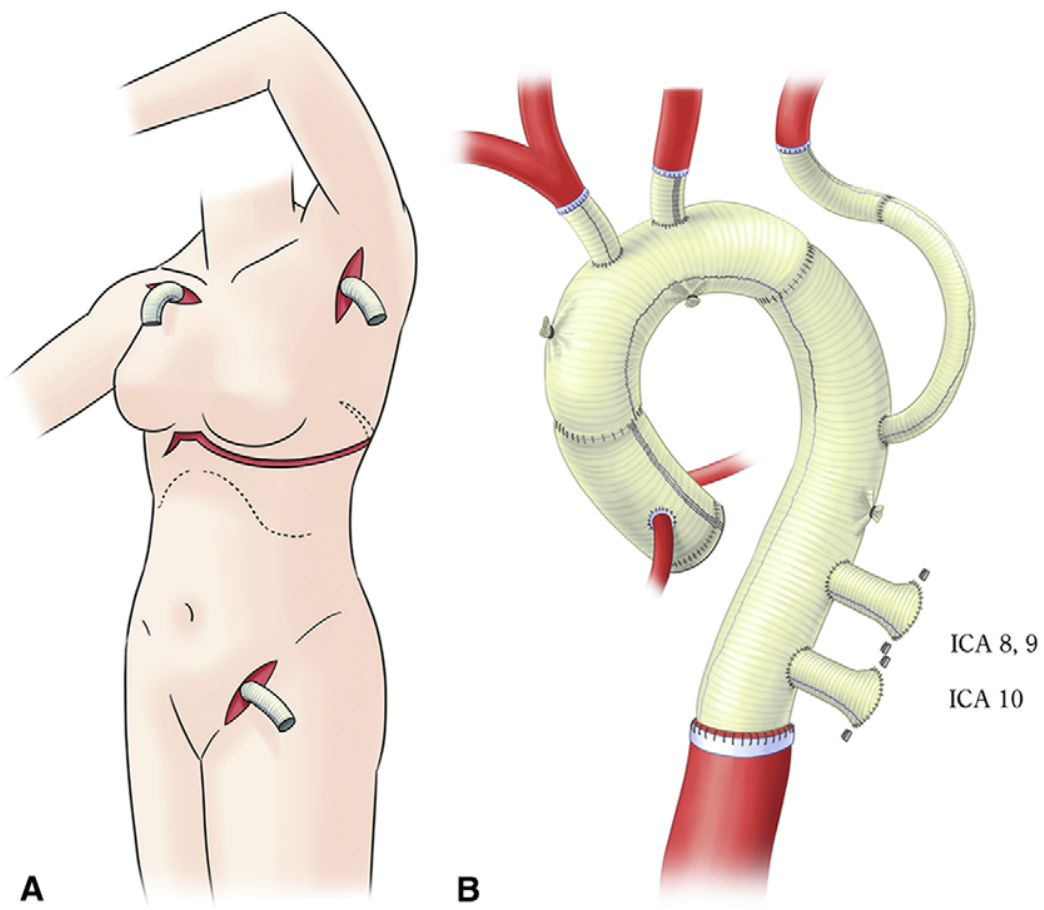

FIGURE 2. Schematic diagrams illustrating operative technique. A, Prosthetic vascular grafts were anastomosed to sides of left axillary, right subclavian, and left femoral arteries. Bilateral anterior thoracotomy through fourth intercostal space with limited incision on right side, transverse sternotomy, transection of fifth and sixth ribs posteriorly, and further extension of sixth intercostal space were used to expose entire thoracic aorta. B, Final appearance of replaced thoracic aorta, with reconstruction of eighth, ninth, and tenth intercostal arteries (ICA). 
extubated on postoperative day 4 and subsequently sent back to the local hospital for rehabilitation. Postoperative 3-dimensional CT showed that the thoracic aorta, almost all of which had been replaced, to be in good condition and demonstrated that the reconstructed intercostal arteries were patent.

\section{DISCUSSION}

The cardinal features of Marfan syndrome involve complex cardiovascular pathology together with variable degrees of chest deformity. ${ }^{1}$ Pectus excavatum and scoliosis in their extreme forms can cause restrictive respiratory dysfunction, ${ }^{2}$ even after the successful repair of aortic lesions. Another reportedly unique respiratory complication is tracheal compression, which is associated with innominate artery aneurysm with concurrent mild chest deformity. ${ }^{3}$ In our case, respiratory distress was a consequence of the PVO, as detected in the CT scans. To the best of our knowledge, PVO caused by extensive aortic aneurysm has not been previously reported in a patient with Marfan syndrome. We believe that the PVO was caused by compression of the right pulmonary veins between the rapidly expanding dissecting descending thoracic aorta and enlarged aortic root in the context of pectus excavatum and scoliosis of the thorax. The PVO observed was also attributable to the hemodynomic effects of the aortic regurgitation.
The surgical technique best used for the treatment of extensive thoracic aortic aneurysm has not yet been established. A staged approach with the elephant trunk technique tends to be favored at our institution when the ascending aorta, the arch, and the descending thoracic or thoracoabdominal aorta are involved. In this case, however, a single-stage approach was indicated for the following reasons. First, if replacement of the descending thoracic aorta had been performed in an initial operation, crossclamping the transverse aortic arch would have led to serious hemodynamic deterioration through significant aortic insufficiency. Second, a single-stage approach eliminated the risk of descending thoracic aortic rupture during the interval between the first stage (aortic root and arch replacement) and the second stage (descending thoracic aortic replacement). ${ }^{4}$

\section{References}

1. Milewicz DM, Dietz HC, Miller DC. Treatment of aortic disease in patients with Marfan syndrome. Circulation. 2005;111:e150-7.

2. Adachi I, Ogino H, Imanaka H, Matsuda H, Minatoya K, Sasaki H. Aortic root replacement in a patient with pulmonary dysfunction caused by severe chest deformity associated with Marfan syndrome. J Thorac Cardiovasc Surg. 2005;130: 213-5.

3. de Feiter PW, van Mook WN, Jacobs MJ. Tracheal compression caused by an innominate artery aneurysm after thoracic aortic aneurysm repair in a patient with Marfan disease. J Thorac Cardiovasc Surg. 2005;129:943-4.

4. Kouchoukos NT, Mauney MC, Masetti P, Castner CF. Single-stage repair of extensive thoracic aortic aneurysms: experience with the arch-first technique and bilateral anterior thoracotomy. J Thorac Cardiovasc Surg. 2004;128:669-75. 